\title{
Design of Warship Simulation Using Variable-Chromosome Genetic Algorithm
}

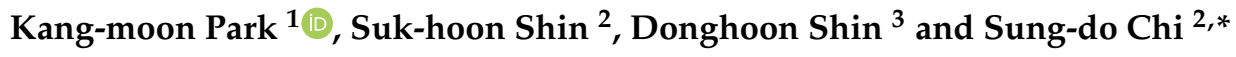 \\ 1 Department of Computer Science, College of Natural Science, Korea Naval Academy, Changwon-si 51704, \\ Korea; kmun422@naver.com \\ 2 Department of Software, Korea Aerospace University, Goyang-si 10540, Korea; ev4shin@naver.com \\ 3 Department of Mechanical Systems Engineering, Sookmyung Women's University, Seoul 04310, Korea; \\ dhshin@sookmyung.ac.kr \\ * Correspondence: sdchi@kau.ac.kr; Tel.: +82-2-300-0184
}

Received: 5 September 2019; Accepted: 1 October 2019; Published: 2 October 2019

check for updates

Featured Application: A variable-chromosome genetic algorithm, expected to overcome the difficulty of a warship simulation in a complex situation.

\begin{abstract}
A genetic algorithm (GA) is a global search algorithm based on biological genetics. GAs are generally used for industrial applications, artificial neural networks, web applications, the defense industry, and so on. However, it is difficult to apply GAs to more complex situations because of the fixed number of chromosomes. In this research, in order to overcome this limitation, we propose a variable-chromosome GA with a chromosome attachment feature. Verification of the algorithm is carried out through anti-submarine high value unit (HVU) escort mission simulations. Ultimately, it is confirmed that the GA using the variable chromosome is more effective in dealing with highly complex missions, whereby the number of chromosomes gradually increases.
\end{abstract}

Keywords: variable-chromosome genetic algorithm; warship simulation; M\&S; multi-agent system

\section{Introduction}

A Genetic Algorithm (GA) is a parallel, global search algorithm based on the principles of natural selection and biological genetics. A great deal of research has been carried out on the theories and techniques regarding the evolutionary strategies and genetic programing related to biological evolutionary processes-namely, natural selection and genetic rules. Particularly for GAs, a significant amount of simulation-based research has been directed toward industrial uses, artificial neural networks (ANNs) [1], and web applications that require optimal solution search algorithms [2]. In addition, because of the usability of highly-autonomous battle object modeling on agent-based modeling and simulation (M\&S) systems, research has been conducted on tactics generation using agent-based M\&S systems and Gas [3-6].

However, due to the operating circumstances of the system, the number of chromosomes utilized in previous research studies has been fixed, which has thereby limited the number of situations that can be generated by the GA. Another difficulty is that the tactics must be optimized using a restricted number of rules. Since the number of rules included in agents does not need to vary, a fixed chromosome GA is quite useful in stable situations.

In this paper, to improve upon these deficiencies, a GA using a chromosome attachment is proposed. This constitutes a new genetic operation that offers variability in terms of the number of chromosomes. The proposed algorithm is then applied to anti-submarine high value unit (HVU) escort mission simulations [7], in order to confirm its effectiveness. 
This paper is organized as follows: In Section 2, backgrounds of the research are presented. The descriptions for the proposed variable-chromosome GA are discussed in Section 3. The GA-based simulation methodology using the variable chromosome is demonstrated in Section 4. In addition, a case study is conducted to verify the simulations in Section 5. In Section 6, conclusions and future research are discussed.

\section{Backgrounds}

\subsection{Genetics: Biological View-Point}

Trial and error processes are an essential part of evolution [8]. These processes are encountered through mutations, however these mutations do not adapt to the environment. Nevertheless, the theory of evolution predicts that evolution occurs when high rates of mutation appear in a specific situations [9-11]. That is, mutation is an essential process from an evolutionary point of view. In addition, gene and chromosome mutations occur within the overall mutation process. The chromosome attachment (or disjunction) phenomenon is a type of chromosome mutation. Chromosome attachment in real life causes conditions such as Down syndrome [12] and Turner syndrome [13], but it can also bring changes in the number of chromosomes in an evolutionary process. It has been established that chromosome changes are an important component of evolutionary processes. However, GAs that take advantage of these evolutionary mechanisms are limited, in that they usually only use fixed chromosomes.

\subsection{Conventional GA-based Simulations}

Significant research has been conducted on the use of GAs for industrial applications, ANNs, and web applications where optimal solution search algorithms are required. Studies utilizing GAs to optimize industry-related problems are continually being produced. These studies are generally focused on energy management problems [5] and building performance analyses [6]. In addition, ongoing research efforts are applying GAs to national defense M\&S systems [2,3].

These studies use GAs to solve problems in various fields. However, they only solve problems in fixed environments because they rely on classical GAs using fixed chromosomes. Cross-species evolution cannot be observed because the number of chromosomes is fixed. To solve this problem, research has been carried out to improve GA performance, especially with variable-length chromosomes [5,6,14], and Jumping Gene Genetic Algorithms (JGGA) [15,16].

A variable-length chromosomes algorithm allows a chromosome to create a child between a different number of rules. For example, if there are five rules in parent A chromosome, and seven in parent $B$ chromosome, an operating child can still be created between the two, through a cross-over operation. In a similar fashion to finding the shortest path in a graph, the algorithm is useful when children with a varying length chromosome occur. However the operation only makes cross-over possible, it does not mean it can increase or decrease the number of rules.

On the other hand, the JGGA is the genetic algorithm based on a jumping gene, which, by its definition, can be found in the genetic mechanism. This entails a certain type of gene, in one chromosome, which jumps into another location of that chromosome, or into a different chromosome. The name comes after its behavioral feature, in that the gene seems to be jumping to another chromosome. This research uses copy-and-paste and cut-and-paste operation to code jumping genes. In the end, a better search value has been yielded, as the algorithm has produced various types of children.

\section{Proposed Variable Chromosome}

In this paper, we propose a variable-chromosome GA to overcome the problems inherent with fixed-chromosome GAs. The variable-chromosome GA has a variable number of chromosomes for one individual undergoing generation change [1]. Due to changes in the number of chromosomes of an individual, it is possible for the GA to evolve and adapt to situations that are more complex than 
initially expected. Also, it becomes possible to adapt to tactics that require greater complexity and more chromosomes, as shown in Figure 1. The composition is similar to a conventional GA, however, a new genetic operation called attachment $[17,18]$ is added to give the chromosome variability. The details of attachment are dealt with in Section 3.2.

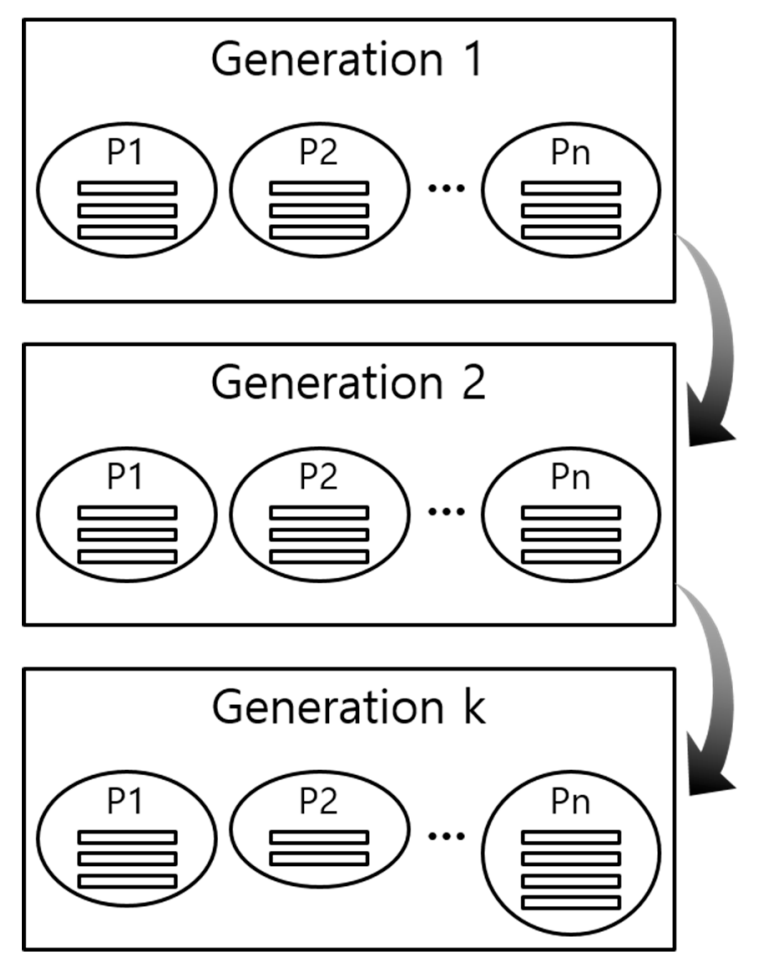

Figure 1. The change of the number of chromosomes.

\subsection{Chromosome Structure}

A chromosome is organized into many genes, as shown in Figure 2. Each of the genes is a unit and has a mean. For example, a chromosome organization can have many genes. These organized chromosomes make an individual (chromosome set).

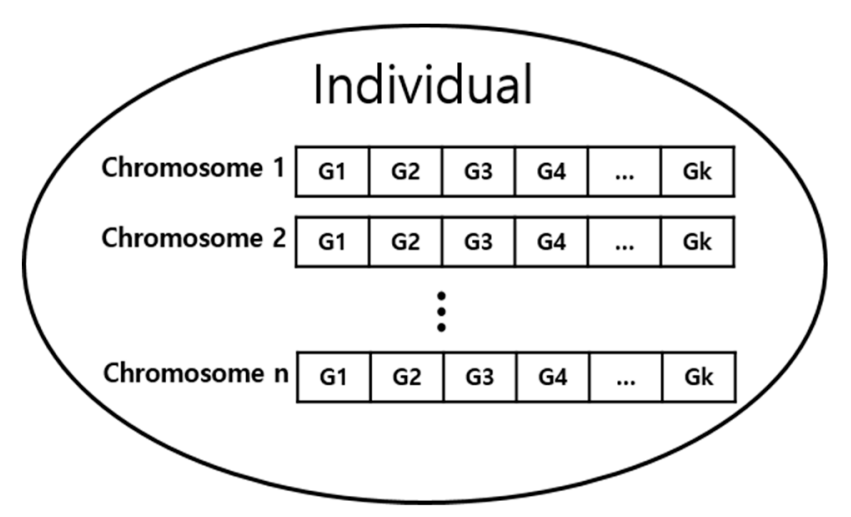

Figure 2. Chromosome structure.

\subsection{Genetic Operation}

Genetic operations in evolution with GAs using variable chromosomes are the cross-over and mutation factors that are used in conventional GAs, however, without using the fixed chromosomes and attachment that are newly proposed in this paper. Cross-overs and mutations are used in classic fixed-chromosome GAs; however, the newly added attachment in this study is a chromosome mutation 
mechanism that makes it possible to change the number of chromosomes. These three operations are executed during cross-breeding after a generation.

The cross-over plays a role in exchanging the chromosomes of the parents from a random point. This point is called a cross-over point. Through this process, the offspring take chromosomes from their parents equally. Mutation denotes that part of a parent's chromosome has changed.

Figure 3 shows the chromosome attachment process. Chromosome attachment is a function in which a randomly selected chromosome attaches to another chromosome during cross-breeding. Through this process, the number of chromosomes of a population can be changed, and this allows for adaptation to a complex situation.

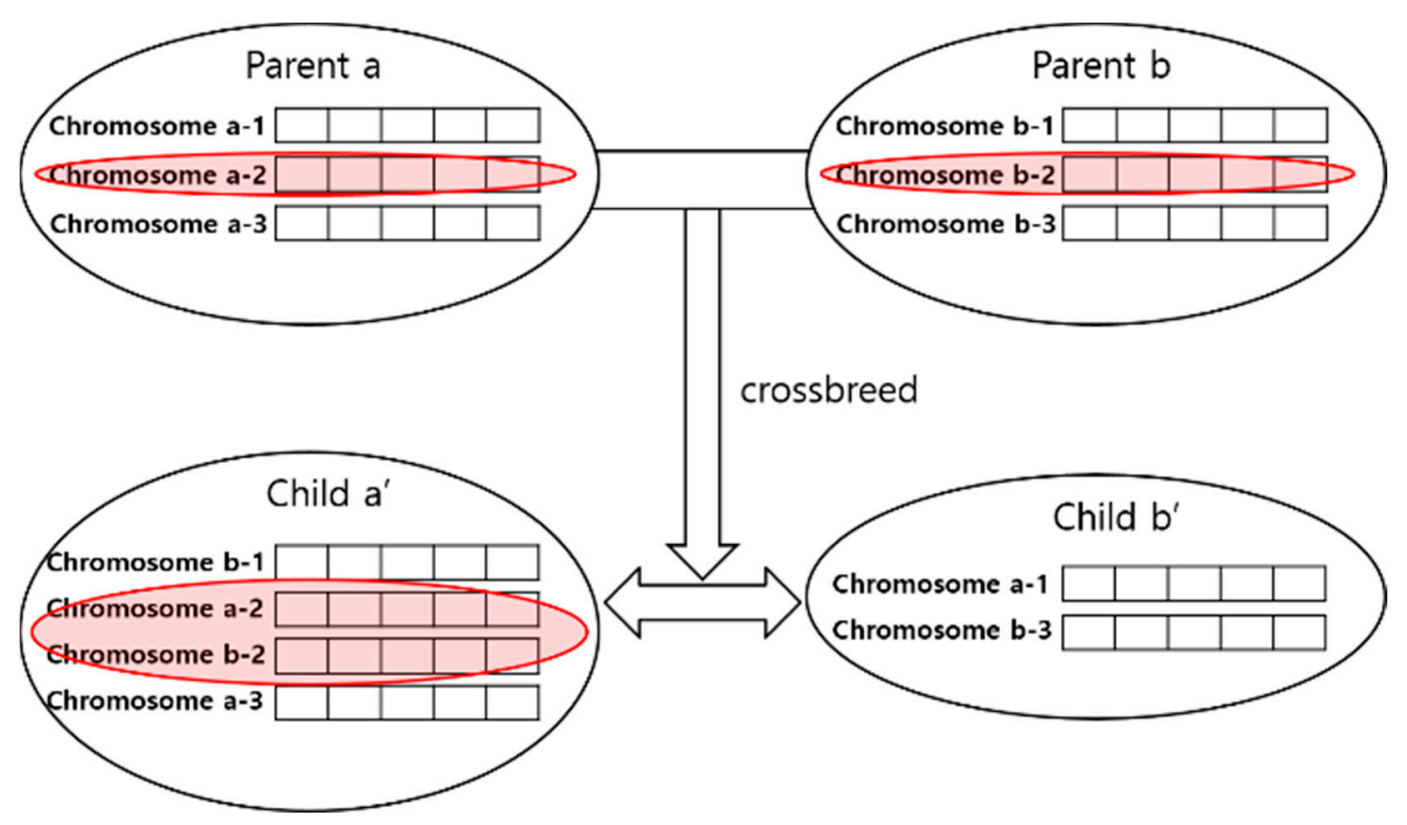

Figure 3. Proposed genetic operation: attachment.

\subsection{Simulation Methodology}

To generate chromosomes loaded on a simulation model, a GA is designed and implemented so that it may be applied to an anti-submarine HVU escort mission simulation. The GA is operated via three phases, as depicted in Figure 4. The operation process is the same as in the conventional approach, except for the genetic operations. 


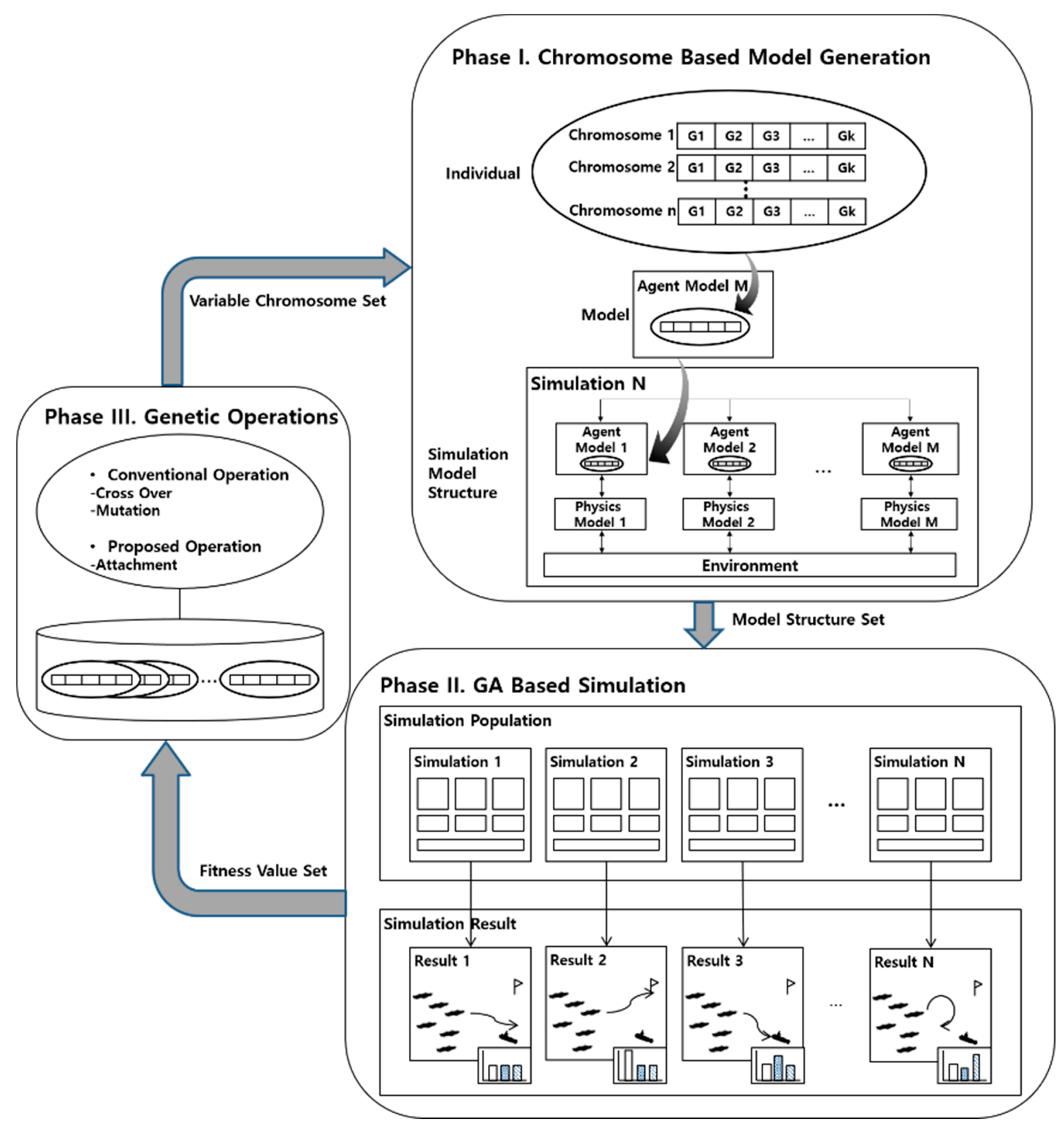

Figure 4. Overall methodology.

Phase 1: Chromosome agent model architecture

Figure 2 shows the chromosomes organized by genes. These chromosomes, which are loaded on each model, are composed of 10 random chromosomes for the first generation. There are 10 chromosomes in an individual for the first generation, however, more or less than 10 chromosomes can be implemented via attachment after some generations have passed. Accordingly, it is possible to achieve flexible evolution, as the number of chromosomes is increased or decreased as necessary.

Phase 2: GA-based simulation

A generation in a GA-based simulation has a total of $\mathrm{N}$ simulations, as shown in Figure 4. Each simulation enables different movement and results, due to the chromosomes loaded on the model. In addition, the fitness value from the simulation results influences the next generation in the simulation.

Phase 3: Fitness values, and generating the next population using the genetic operations 
In the third phase, populations are first sorted by their fitness values. Subsequently, new populations are created via the genetic operations during the cross-breeding of generations, as stated in Section 3.2. In particular, the number of chromosomes can vary with the attachment operation. These three genetic operations are carried out for some parts of the chromosomes, via a set probability, which puts the emphasis on carrying forward a better population to the next generation.

\section{Case study: Optimal Tactics Generation}

To validate the proposed methodology, we tested an anti-submarine HVU escort mission simulation. A submarine has firepower that is almost equal to a fleet. Thus, there are many warships that are required to cope with submarines [7]. In this case study, we allocate warships, an HVU, helicopters, false targets, and an enemy submarine to create a complex warfare environment, as shown in Figure 5. The false targets are allocated randomly in every simulation, however, the warships, HVU, helicopters, and enemy submarine are allocated at fixed positions.

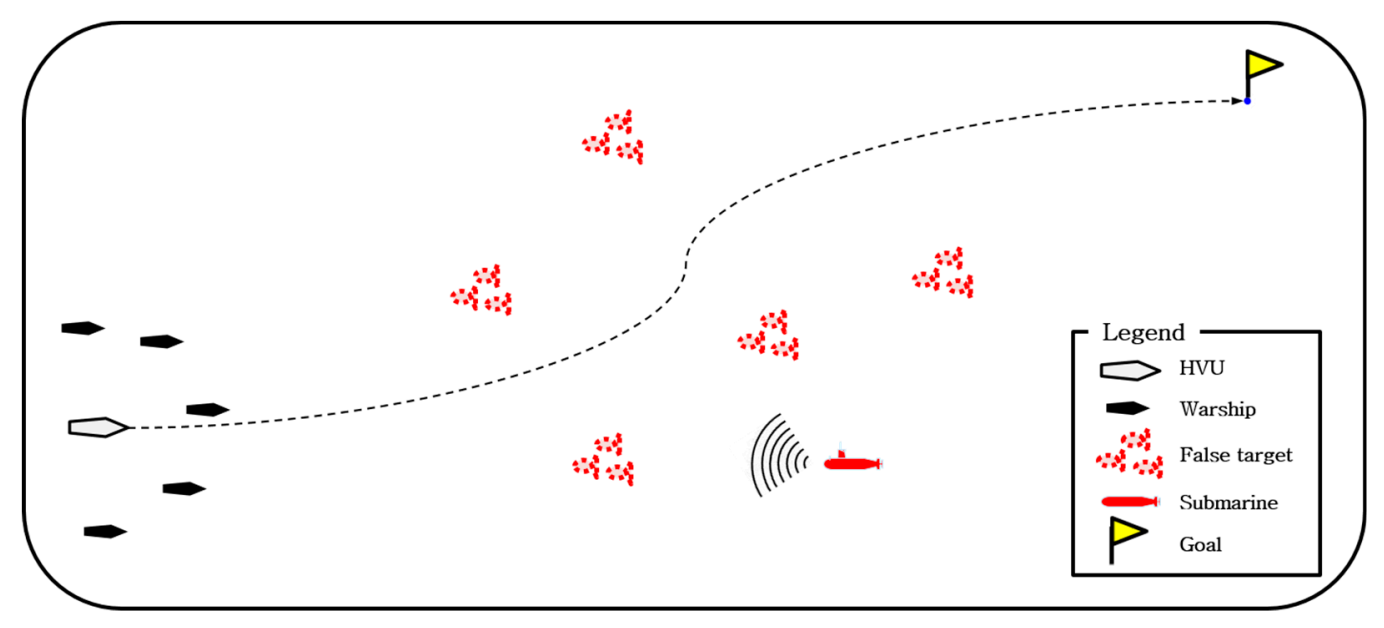

Figure 5. Mission scenario.

If the HVU arrives at its goal, then the mission is considered to be successful. The warships take warning and escort the HVU, surrounded by reconnaissance, to protect it from destruction. The warships attempt to detect the false targets and the enemy submarine while moving to the goal. If the enemy submarine is detected, a battle may arise. However, if it is a false target, the warships can ignore it and move ahead. The helicopters attempt to identify and classify the false targets and enemy submarine. The false targets do nothing at their randomly fixed positions, and the warships are not able to separate the false targets from the enemy submarine before the objects are classified. However, the enemy submarine is not influenced by the false targets. In addition, if the enemy submarine detects a warship or HVU, it will attack immediately.

\subsection{Initialization}

The number of generations in the evolution governed by the GA is set to a total of 10, because there are almost no changes after the seventh generation. The number of existing populations in a generation is set to 50 , because there are almost no differences of more than 50 . The 50 populations have 10 rules (chromosomes) in the first generation. Subsequently, decisions are made as to which populations survive to the next generation, and breeding takes place depending on the fitness values. Five groups survive to the next generation.

- The top 10 fitness value populations from the previous generation are determined. 
- The 10 offspring are created from breeding between the best fitness value population and a random selection from the top 10 fitness value populations from the previous generation.

- The 10 offspring are created from breeding between the second best fitness value population and a random selection from the top 10 fitness value populations from the previous generation.

- The 10 offspring are created from breeding between two random selections from the top 10 fitness value populations from the previous generation.

- The 10 offspring are created from breeding between two random selections from the top 10 fitness value populations and a random selection from the lowest 10 fitness value populations from the previous generation.

The mutation rate and chromosome attachment rate are set at $10 \%$. The three measures of effectiveness (MOEs) being used for the fitness value are the force survival rate, the enemy damage rate, and the goal achievement rate. Equation (1) shows how the fitness value (f) is determined from the three MOEs. The force survival rate is expressed as the surviving number of warships ( $\mathrm{s}_{\text {ours }}$ ) over the total number of warships ( $\mathrm{n}_{\text {ours }}$ ). The enemy damage rate is expressed as the surviving number of enemy submarines ( $s_{\text {enemy }}$ ) over the total number of enemy submarines $\left(\mathrm{n}_{\text {enemy }}\right)$. The goal achievement rate is expressed as the lineal distance from the HVU to the destination goal at the end of the simulation $\left(\mathrm{d}_{\mathrm{istmoved}}\right)$ over the lineal distance of the HVU to the destination goal at the beginning of the simulation $\left(\mathrm{d}_{\text {isttotal }}\right)$. The sum of the $\omega 1, \omega 2$, and $\omega 3$ weight values must always be 1 .

$$
\mathrm{f}=\omega_{1} \cdot \frac{\text { dist }_{\text {moved }}}{\text { dist }_{\text {total }}}+\omega_{2} \cdot \frac{s_{\text {ours }}}{n_{\text {ours }}}+\omega_{3} \cdot \frac{s_{\text {enemy }}}{n_{\text {enemy }}}
$$

\subsection{Chromosome Agent Modeling}

The warship model is composed of a warship agent model and warship physics model. The warship agent model determines decision making. The warship physics model delivers the required information, or performs a received order. The enemy submarine model is composed of a submarine agent model and a submarine physics model, with the same roles given as in the warship model.

\subsection{Simulation Tests}

To verify the variable-chromosome GA applied attachment, a simulation was carried out. To determine differences stemming from the complexity of the tactics, we varied the fitness value, because tactics complexity differs greatly according to the set fitness value. $\omega 1$ was 0.4 , because it was essential to move toward the goal. Then $\omega 2$ and $\omega 3$ were changed from 0 to 0.6 . The mission complexity was assigned a value from 0.00 to 1.00 , depending on the size of the $\omega 3$.

\section{Discussion}

Figure 6a shows the results of the simulations, with 50 populations and 10 generations in which the fitness value was varied in order to confirm mission complexity differences. In the case with low mission complexity, tactics evolved that focused on evasion and reaching the final goal. These tactics stemmed from the priority being given to surviving. As a result, the average mission success rate converged to almost 0.17 after the fourth generation, of both the GA using the fixed chromosome and the GA using the variable chromosome. This similarity occurred because convergence with low tactics complexity is easy without reference to the number of chromosomes. 


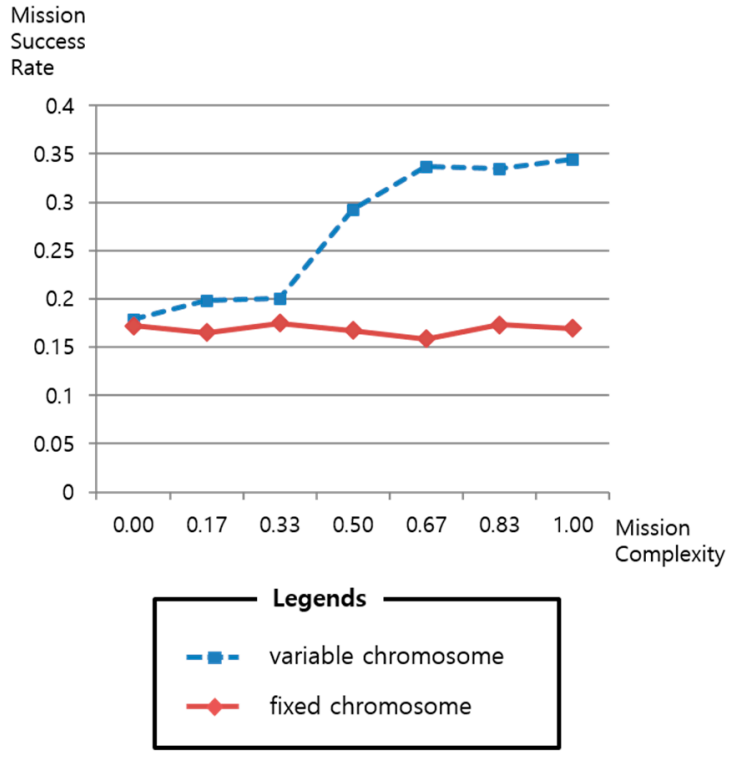

(a)

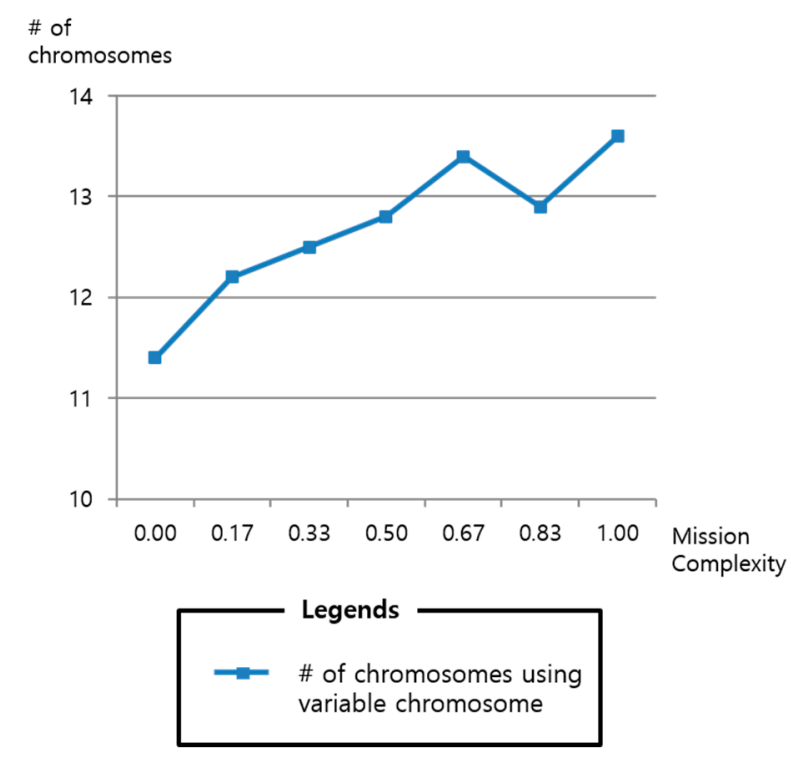

(b)

Figure 6. Simulation results. (a) Mission success rate, (b) The number of chromosomes.

However, in missions with a complexity value of at least 0.5 , the mission success rate of the fixed-chromosome GA converged to 0.17 at the third generation, however, the mission success rate of the variable-chromosome GA converged to almost 0.34 . In addition, when comparing how the tactics evolved, it was observed that the fixed-chromosome GA simply moved toward the goal while the variable-chromosome GA developed more complex offensive tactics, such as detecting, classifying, and attacking the enemy. However, the final mission success rate was only 0.34 , because the difference between the physical specifications of the submarine and the surface ships was too large.

These results occurred because with the fixed-chromosome GA, the limit on the number of rules meant that adaption could not take place for complex tactics, such as aggressive maneuvers. Yet, with the variable-chromosome GA, adaption took place for complex tactics. This shows that the variable-chromosome GA was able to evolve to create more outstanding tactics.

Figure $6 \mathrm{~b}$ is a graph comparing the number of chromosomes in accordance with the mission complexity. It represents the average of the top 10 of the final evolutions of each individual mission complexity. When the mission complexity was low, it was not necessary to evolve complex tactics, and so the number of chromosomes hardly increased. However, when the mission complexity was high, the number of chromosomes increased to almost 14 to adapt to the complex situation.

Figure 7 shows the evolution among a different number of dummies in multiple simulations. As shown in the graph, the fitness value seems to be increasing as each generation evolves, regardless of the number of dummies in a simulation. However, it has a tendency to slow down its increasing rate of fitness value with more dummies, whereupon after the 4 th or 5 th generation, it shows little difference in curves. Figure 8 shows the changes in the number of chromosomes in every generation, in correlation to the number of dummies. The curve shows a relatively steep gradient in simulations with less than two dummies, yet, it appears converged if it involves more than three dummies. In an analysis of the final state, that no longer has any meaningful changes in the number of chromosomes, the research shows that it has evolved into evading the battles, while persisting toward the objective. It chooses rather simpler tactics without increasing the number of chromosomes in complex situations. It is speculated that the limitations have been induced due to the insufficient sets of rules in the simulations. 


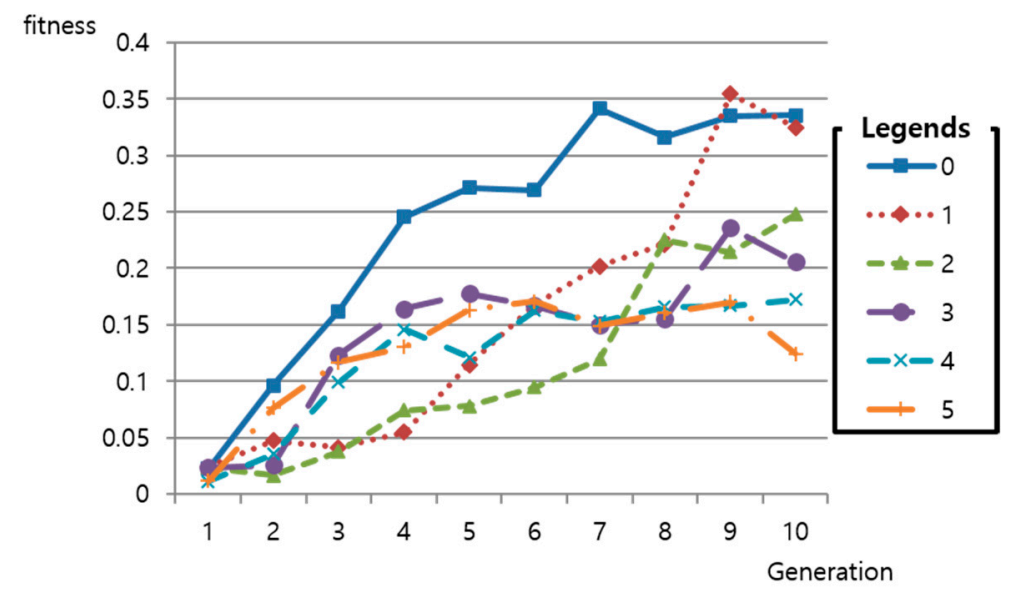

Figure 7. The evolution among different numbers of dummies.

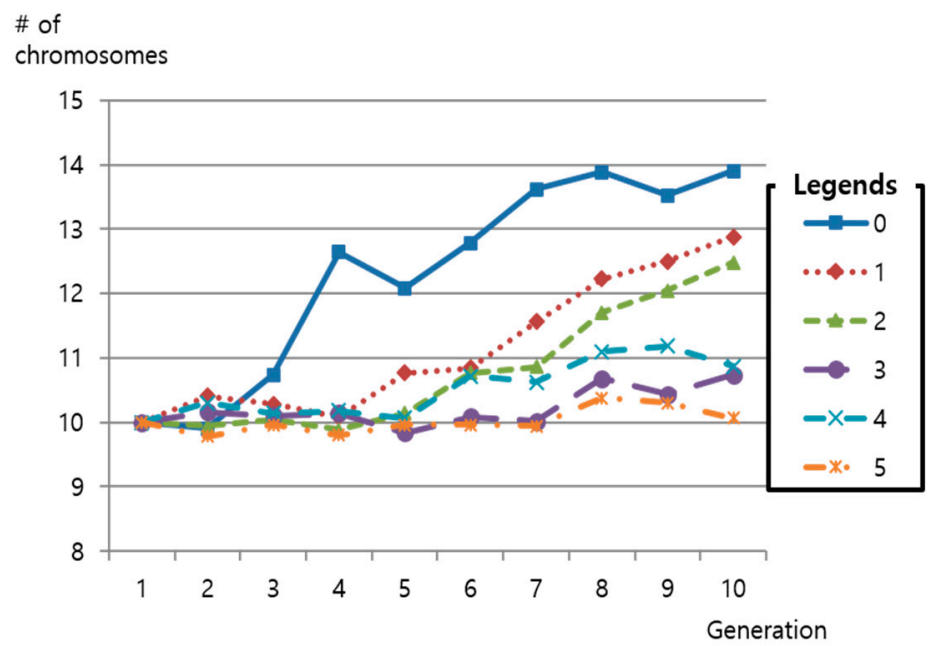

Figure 8. The changes in the number of chromosomes in every generation, and the number of dummies.

\section{Conclusions}

Conventional GAs suffer from a disadvantage; they cannot evolve to an optimal status when faced with unexpected/complex situations, as they are limited by their number of chromosomes. In this paper, a variable-chromosome GA using a chromosome attachment was proposed to overcome this disadvantage. Simulations were carried out for an anti-submarine HVU escort mission. The variable-chromosome GA was applied to these simulations in order to verify the performance capabilities of the algorithm.

The experimental results demonstrated that when varying the fitness value to explore differences in mission complexity, there were no differences between the fixed-chromosome GA and the variable-chromosome GA for simple tactics. However, for complex tactics, the fixed-chromosome GA was not able to achieve a mission success rate above a certain level because of the limitations placed on the number of chromosomes. On the other hand, the simulation using the variable-chromosome GA was able to evolve and ultimately achieve a higher mission success rate, because of the availability of a varying number of chromosomes. These simulations demonstrated the importance of changing the number of chromosomes during complex situations. Furthermore, the research found that complex situations using dummies are not able to achieve the desired evolution that increases the number of chromosomes. It is presumed that this inability results from the simplicity in the sets of the rules, requiring further research for accurate results. 
In the future, research will also be conducted on the potential variable-chromosome GA applications, such as in the application of the structural learning of artificial neural networks. It can also be applied to neural architecture searches (NAS), and automated machine learning (AutoML).

Author Contributions: Conceptualization, K.-m.P. and S.-d.C.; methodology, K.-m.P.; software, K.-m.P.; validation, K.-m.P. and S.-h.S.; formal analysis, S.-h.S. and D.S.; investigation, K.-m.P.; resources, S.-d.C.; data curation, K.-m.P.; writing, original draft preparation, K.-m.P.; writing, review and editing, S.-h.S., D.S. and S.-d.C.; visualization, K.-m.P.; supervision, S.-d.C.; project administration, K.-m.P.; funding acquisition, S.-d.C.

Funding: This research received no external funding.

Conflicts of Interest: The authors declare no conflicts of interest.

\section{References}

1. Park, K.; Shin, D.; Chi, S. Variable Chromosome Genetic Algorithm for Structure Learning in Neural Networks to Imitate Human Brain. Appl. Sci. 2019, 9, 3176. [CrossRef]

2. Li, Z.; Liu, J. A multi-agent genetic algorithm for community detection in complex networks. Phys. A: Stat. Mech. Appl. 2016, 449, 336-347. [CrossRef]

3. Jung, C.H.; Ryu, H.-E.; You, Y.J.; Chi, S.D.; Kim, J.I. Many-to-Many Warship Combat Tactics Generation Methodology Using the Evolutionary Simulation. J. Korea Soc. Simul. 2011, 20, 79-88. [CrossRef]

4. You, Y.J.; Chi, S.D.; Kim, J.I. Simulation-Based Tactics Generation for Warship Combat Using the Genetic Algorithm. IEICE Trans. Inf. Syst. 2011, 94, 2533-2536. [CrossRef]

5. Arabali, A.; Ghofrani, M.; Etezadi-Amoli, M.; Fadali, M.S.; Baghzouz, Y. Genetic-algorithm-based optimization approach for energy management. IEEE Trans. Power Delivery 2012, 28, 162-170. [CrossRef]

6. Nguyen, A.T.; Reiter, S.; Rigo, P. A review on simulation-based optimization methods applied to building performance analysis. Appl. Energy 2014, 113, 1043-1058. [CrossRef]

7. Park, K.M.; Lee, E.B.; Shin, S.H.; Han, S.; Chi, S.D. Modeling and Simulation for Anti-submarine HVU Escort Mission. J. Korea Soc. Simul. 2014, 23, 75-83. [CrossRef]

8. Wright, S. The Roles of Mutation, Inbreeding, Crossbreeding, and Selection in Evolution; Proceedings of the VI International Congress of Genetics; Brooklyn Botanic Garden: Brooklyn, NY, USA, 1932; Volume 1, pp. 356-366.

9. Leigh, E.G., Jr. Natural selection and mutability. Am. Nat. 1970, 104, 301-305. [CrossRef]

10. Ishii, K.; Matsuda, H.; Iwasa, Y.; Sasaki, A. Evolutionarily stable mutation rate in a periodically changing environment. Genetics 1989, 121, 163-174. [PubMed]

11. Taddei, F.; Radman, M.; Maynard-Smith, J.; Toupance, B.; Gouyon, P.-H.; Godelle, B. Role of mutator alleles in adaptive evolution. Nature 1997, 387, 700. [CrossRef] [PubMed]

12. Potter, H. Review and hypothesis: Alzheimer disease and Down syndrome-chromosome 21 nondisjunction may underlie both disorders. Am. J. Hum. Genet. 1991, 48, 1192. [PubMed]

13. Greaney, J.; Wei, Z.; Homer, H. Regulation of chromosome segregation in oocytes and the cellular basis for female meiotic errors. Hum. Reprod. Update 2017, 24, 135-161. [CrossRef] [PubMed]

14. Harvey, I. The SAGA cross: The mechanics of recombination for species with variable length genotypes. In Proceedings of the PPSN, Citeseer, Brussels, Belgium, 28-30 September 1992.

15. Srikanth, R.; George, R.; Warsi, N.; Prabhu, D.; Petry, F.E.; Buckles, B.P. A variable-length genetic algorithm for clustering and classification. Pattern Recognit. Lett. 1995, 16, 789-800. [CrossRef]

16. Juang, C.F. Temporal problems solved by dynamic fuzzy network based on genetic algorithm with variable-length chromosomes. Fuzzy Sets Syst. 2004, 142, 199-219. [CrossRef]

17. Li, Y.Y.; Yeh, E.; Hays, T.; Bloom, K. Disruption of mitotic spindle orientation in a yeast dynein mutant. Proc. Natl. Acad. Sci. USA 1993, 90, 10096-10100. [CrossRef] [PubMed]

18. Sanchez-Perez, I.; Renwick, S.J.; Crawley, K.; Karig, I.; Buck, V.; Meadows, J.C.; Franco-Sanchez, A.; Fleig, U.; Toda, T.; Millar, J.B. The DASH complex and Klp5/Klp6 kinesin coordinate bipolar chromosome attachment in fission yeast. EMBO J. 2005, 24, 2931-2943. [CrossRef] 